\title{
Cancelled project spurs debate over geoengineering patents
}

\section{SPICE research consortium decides not to field-test its technology to reflect the Sun's rays.}

\section{BY DANIEL CRESSEY}

$\mathrm{T}$ echnologies to keep Earth cool could one day provide a radical fix for climate change - and, in a world struggling to control its greenhouse-gas emissions, could also prove highly lucrative for inventors.

But should individual researchers, or companies, be allowed to own the intellectual property (IP) behind these world-changing techniques? The issue was thrust into the spotlight last week after a controversial geoengineering field trial was cancelled amid concerns about a patent application by some of those involved in the project, as first reported by Nature ${ }^{1}$.

The £1.6-million (US\$2.5-million) Stratospheric Particle Injection for Climate Engineering (SPICE) project was funded by the UK government to investigate whether spurting reflective aerosols into the stratosphere could help to bounce some of the Sun's warming rays back into space. As part of this project, SPICE had planned to test a possible delivery system: pumping water up a 1-kilometre-long hose to a balloon, where it would be sprayed into the sky.

The project had already sparked protests from environmentalists wary of geoengineering $^{2}$. But "a potentially significant conflict of interest" over a patent application for SPICE's technology, which some team members only recently became aware of, was a decisive factor in the cancellation, says project leader Matthew Watson, an Earth scientist at the University of Bristol, UK. The patent was submitted by Peter Davidson, a consultant based on the Isle of Man who was an adviser at the workshop that gave rise to SPICE, and Hugh Hunt, an engineer at the University of Cambridge, UK, who is one of the SPICE project investigators.

UK funding bodies require anyone assessing or applying for grants to declare relevant

potential conflicts of interest. Davidson and Hunt say that they were clear about their patent application before SPICE was awarded funding, and there is no suggestion that they acted inappropriately. But at least one of the funding councils is now investigating the circumstances surrounding the SPICE grant, and the patent in question, says Watson.

Hunt blames a culture clash for the confusion. "It is completely normal for engineering projects to be protected by IP," he says. "The issue here is that in climate science there is mistrust of IP, and I understand that now." He says he does not expect to earn any money from the patent.

SPICE's climate modelling and other technology development work will continue, but the incident is another blow for a field already troubled by concerns over governance. In 2010, researchers and policy-makers gathered

"The issue here is that in climate science there is mistrust of IP." at the Asilomar Con-

ference Center near Monterey, California, to agree a set of guiding principles for the field - an effort that largely failed ${ }^{3}$.

A smaller group had already produced the 'Oxford Principles', stating that geoengineering should be "regulated as a public good". The lead authors of those principles have warned that patenting of geoengineering technologies could "have serious negative impacts", by creating a culture of secrecy that could delay much-needed developments.

Climate scientist David Keith of Harvard University in Cambridge, Massachusetts, agrees, advocating legal restrictions on patents related to solar-radiation management. Any technologies that could be controlled by a small number of people, yet have the capacity to rapidly alter our planet's climate, "are deeply troubling", he says. But Keith is not against patenting in principle - he has applied for patents on techniques to remove carbon dioxide directly from the atmosphere.

Shobita Parthasarathy, a public-policy researcher at the University of Michigan, Ann Arbor, says that the field urgently needs to agree on detailed rules for IP. In 2010, she noted a "dramatically increasing" number of patent applications in the area, containing broad language that could allow a small number of patent holders to take control of a huge swathe of technologies ${ }^{4}$. One possible solution, she says, is to develop a unique system for handling geoengineering patents, akin to the way that atomic-energy patents are controlled in the United States. That system puts certain technologies off-limits, and allows the government to take control of some intellectual property. "I don't think the solution is to get rid of IP," she says.

Another option might be to allow patentholders to receive royalties, but without the option to restrict the use of the patent, says Tim Kruger, a researcher at the Geoengineering Programme, University of Oxford, UK, who helped to develop the Oxford Principles. This would allow some research and development to proceed, while still providing a financial incentive to work in the area, he says.

But geoengineering patents of any kind could give companies a vested interest in the continuation of climate change, argues Holly Buck, a social scientist who has studied the ethics of geoengineering. "It seems conceptually wrong to create conditions for an enterprise that would institutionally benefit from a stressed climate." - SEE EDITORIALP.415

\footnotetext{
1. Cressey, D. Nature http://doi.org/hw2 (2012)

2. Macnaghten P. \& Owen R. Nature 479, 293 (2011).

3. Tollefson, J. Nature 464, 656 (2010).

4. Parthasarathy, S. et al. A Public Good? Geoengineering and Intellectual Property (Univ. Michigan, 2012); available at go.nature.com/scrl4g
}

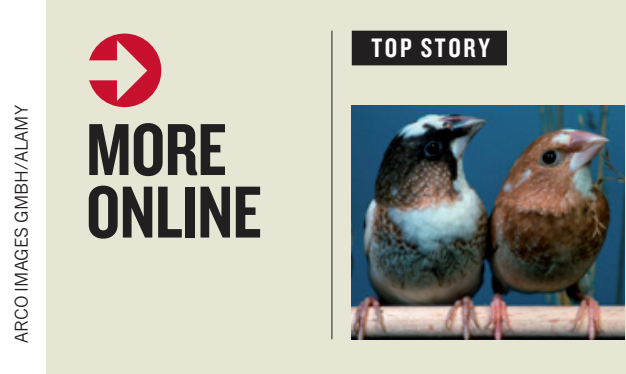

Finches learn even when practice isn't perfect go.nature.com/ vqnilol

\section{MORE NEWS}

- Source found for missing water in sea-level rise go.nature.com/damx $2 \mathrm{~m}$ - Why great ideas tend to come when you aren't trying go.nature.com/ ekb8rh

- Volcanic eruptions trigger shocking finding go.nature.com/falzm3

\section{ON THE BLOG}

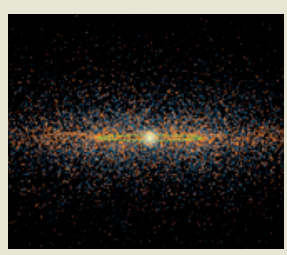

Tally of most hazardous asteroids doubles go.nature.com/ iynpgq 